\title{
Corrigendum and Addendum \\ to \\ « Multifunctions on Abstract Measurable Spaces and Applications to Stochastic Decision Theory 》 (*) (**).
}

\author{
C. J. HtMmelbeRG - F. S. VAN Vleck (Lawrence, Kansas)
}

Summary. - This note points out that the "only if" part of Theorem $\mathbf{1}^{\prime}$ (i) of the above paper [Annali di Matematica pura ed applicata, (IV), 101 (1974), pp. 229-236] is false as is part (ii) of Theorem 2. Counterexamples are given and a weak form of the "only if " part of Theorem 1' (i) is established.

The purpose of this note is to point out an error, and its consequences, in the paper [HV-1] mentioned in the title, to give appropriate counterexamples, and to establish a correct, though weaker, result. The notation used here is that of the original paper.

First, the "only if " part of Theorem $\mathbf{1}^{\prime}$ (i) is not true. This part of the theorem asserted that an $(\mathcal{A}, \mathcal{C})$-measurable multifunction $F$ with complete values from an arbitrary measurable space $(T, \mathcal{A})$ to a separable metric space $X$ has a Castaing representation, i.e. there is a countable set $U$ of measurable selectors of $F$ such that $F(t)=\overline{U(t)}$ for all $t \in T$. The "proof " does not follow from the "only if " part of the proof of [C, Theorem 5.4] as we stated. In fact, the following counterexample, due to Nishura [N], shows that the statement is false even if the measurable space $(T, A)$ is complete and $X$ is a Polish space.

Before giving Nishiura's counterexample we should point out that this incorrect result would imply, among other things, that $(\mathcal{A}, \mathcal{C})$-measurability is equivalent to weak measurability under the hypotheses of Theorem $\mathcal{I}^{\prime}(\mathrm{i})$. This result, which is false, has been reported, based upon our error, in the survey by D. WAGNER [W, Theorem $4.2(e)(x i \Rightarrow i x)]$. Another consequence would be that the intersection of countably many closed-valued, weakly measurable multifunctions is weakly measurable. That too is false; a counterexample will be included in [HPV].

EXAMPLE 1 [Nishiura]. - Let $T$ be the set of irrational numbers and let $A \in A$ iff $A$ is residual (cf. [K, p. 201]) in $T$ or coresidual in $T$. Let $\mu(A)=1$ if $A$ is residual

(*) Entrata in Redazione il 16 novembre 1977.

(**) Partially supported by National Science Foundation Grant MCS 76-24436. 
and $\mu(A)=0$ if $A$ is coresidual. Then $(T, \mathcal{A}, \mu)$ is a finite, complete measure space. Let $X=T$ and let $F: T \rightarrow X$ be the identity function. Then $F^{-1}(K)$ is coresidual for each compact $K \subset X$ and hence $F$ is $(\mathfrak{A}, \mathrm{C}$ )-measurable. But $F$ is not measurable and so can have no measurable selectors and, hence, no Castaing representation.

We can, however, establish the following weaker result if we assume the continuum hypothesis. (Recall from [HV-2] that $F: T \rightarrow X$ is $(\mathcal{R}, \mathcal{C})$ measurable iff $F^{-1}(B) \in \mathcal{R}$ whenever $B$ is compact.)

THEOREM. - Assume the continuum hypothesis. Let $T$ be a set with a $\sigma$-ring $R$, let $X$ be separable metric, and let $F: T \rightarrow X$ be a multifunetion with elosed, nonempty values. If $F$ is $(\mathcal{R}, \mathcal{C})$-measurable, then there is a countable family $U$ of $(\mathcal{R}, \mathcal{C})$-measurable selectors for $F$ such that $F(t)=\overline{U(t)}$ for each $t \in T$.

Proof. - Let $D=\left\{x_{m} \mid m=1,2, \ldots\right\}$ be dense in $X$ and let $B_{m n}=B\left(x_{m}, 1 / n\right)$ be the closed ball of radius $1 / n$ about $x_{m}$ : Let $u_{m n}: T \rightarrow X$ be an $(\mathcal{R}, \mathcal{C})$-measurable selector for $F$ such that $u_{m n}(t) \in B_{m n}$ whenever $B_{m n} \cap F(t) \neq \emptyset$. (That such functions $u_{m n}$ exist is established in the lemma below.) Then $U=\left\{u_{m n} \mid m, n=1,2, \ldots\right\}$ is the desired family. For let $t \in T, x \in F(t)$ and $\varepsilon>0$. Choose $n$ and $m$ such that $2 / n<\varepsilon$ and $d\left(x_{m}, x\right)<1 / n$. Then $x \in B_{m n} \subset B(x, \varepsilon)$ and $u_{m n}(t) \in B_{m n} \cap F(t) ;$ so $d\left(u_{m n}(t), x\right)<\varepsilon$. Thus $U(t)$ is dense in $F(t)$.

LEMma. - Assume the continuum hypothesis. Let $B$ be a closed ball in a separable metric space $X$, let $(T, \mathcal{R})$ be a set with a $\sigma$-ring $\mathcal{R}$, and let $F: T \rightarrow X$ be an $(\mathcal{R}, \mathcal{C})$ measurable multifunction with closed values. Then there exists an $(\mathcal{R}, \mathcal{C})$-measurable selector $f$ for $F$ such that $f(t) \in B$ whenever $F(t) \cap B \neq \emptyset$.

Proof. - Let $S=F^{-1}(B)$ and define $F_{1}: S \rightarrow X$ by $F_{1}(t)=F(t) \cap B$ for each $t \in S$. Then $F_{1}$ is $\left(\Re_{1}, \mathcal{C}\right)$-measurable (where $\Re_{1}=\{A \in R \mid A \subset S\}$ ), since, for each compact $K \subset X, F_{1}^{-1}(K)=F^{-1}(B \cap K)$. Now let $f_{1}: S \rightarrow X$ be an $\left(\Re_{1}, \mathcal{C}\right)$-measurable selector for $F \mid S$, and let $f_{2}: T \rightarrow X$ be an $(\mathcal{R}, \mathcal{C})$-measurable selector for $F$. Define $f: T \rightarrow X$ by

$$
f(t)= \begin{cases}f_{1}(t) & \text { if } t \in S, \\ f_{2}(t) & \text { if } t \in T-S\end{cases}
$$

Then $f$ is the desired selector. It is $(\mathcal{R}, \mathcal{C})$-measurable; for if $K$ is compact, then

$$
f^{-1}(K)=f_{1}^{-1}(K \cap B) \cup f_{2}^{-1}(K \cap(X-B))
$$

which belongs to $R$, since $f_{1}^{-1}(K \cap B) \in \mathcal{R}_{1} \subset \mathcal{R}$ by the compactness of $K \cap B$, and $f_{2}^{-1}(K \cap(X-B)) \in \Re$ by the $\sigma$-compactness of $K \cap(X-B)$.

The "if " part of Theorem $\mathbf{1}^{\prime}(\mathrm{i})$ is true since weak measurability, and hence $(\mathfrak{A}, \mathrm{C})$-measurability, follows from a Castaing representation by measurable func- 
tions. However, the converse of the theorem of this note is not true as the following counterexample shows.

Example 2. - Let $\left\{\boldsymbol{r}_{n} \mid n=1,2, \ldots\right\}$ be the set of non-negative rational numbers, and let $T$ and $X$ be as in Example 1. For each $n$, define a function $u_{n}: T \rightarrow X$ by

$$
u_{n}(t)=t+r_{n} \quad \text { for } t \in T
$$

Define $F: T \rightarrow X$ by

$$
F(t)=\cup\left\{u_{n}(t) \mid n=1,2, \ldots\right\}
$$

Then each $u_{n}$ is $(\mathcal{A}, \mathrm{C})$-measurable, but $F$ is not since, for example, $F^{-1}(\{x\})$ is the segment $(-\infty, x] \cap T$, which is neither residual nor coresidual.

One of the consequences of Theorem $1^{\prime}$ (i) in [HV-1] was Theorem 2 (ii), which asserted that $F_{1}+F_{2}$ is an $(\mathcal{A}, \mathcal{C})$-measurable multifunction when both $F_{1}$ and $F_{2}$ are $(\mathcal{A}, \mathcal{C})$-measurable. This is also false as the following example shows.

EXAMPLE 3. Let $T$ and $X$ be as in Example 1 and embed $X$ as a closed subset of a Banach space $E$ using, for example, the Kuratowski-Wojdyslawski embedding theorem (cf. [Hu, p. 81]). (We can get $X$ closed in $E$, by giving $X$ a complete metric before embedding it in $E$.) Let $F_{1}: T \rightarrow E$ be as in Example 1. Let $0 \neq b \in E$ and define $F_{2}: T \rightarrow E$ by

$$
F_{2}(t)= \begin{cases}-F_{1}(t) & \text { for } t \in T^{-}=\{t \mid t<0\} \\ b-F_{1}(t) & \text { for } t \in T^{+}=\{t \mid t>0\} .\end{cases}
$$

(The algebraic operations in the definition of $F_{2}$ are those of $E$ and not those of $X$ ) Then, for each compact $K \subset E$,

$$
\begin{aligned}
F_{2}^{-1}(K)=\left\{t \mid F_{2}(t) \in K\{\right. & =\left\{t<0 \mid-F_{1}(t) \in K\right\} \cup\left\{t>0 \mid b-F_{1}(t) \in K\right\} \\
& =\left[T^{-} \cap F_{1}^{-1}(-K)\right] \cup\left[T^{+} \cap F_{1}^{-1}(b-K)\right] .
\end{aligned}
$$

Since $K$ compact implies $-K$ and $b-K$ are compact, $F_{2}^{-1}(K)$ is a union of two sets each of which is coresidual since each is a subset of a coresidual set. Thus $F_{2}$ is (At, C)-measurable. The function $F_{1}+F_{2}$ is not $(\mathcal{A}, \mathrm{C})$-measurable since it is the step function whose value for $t<0$ is zero and whose value for $t>0$ is $b$.

REMARK. - This example can not be simplified by regarding the function $F_{1}=F$ : $T \rightarrow X$ of Example 1 as a function from $T$ into $R^{1}$ and then proceeding as in Example 3 with $E$ replaced by $R^{1}$. The trouble is that $F$ is (A,, $\mathcal{C}$ )-measurable as a map into $X$, but not as a map into $R^{x}$.

The remaining theorems in [HV-1] which appear to depend on Theorem $1^{\prime}$ (i) are true. For instance, Theorem 3 (ii) is true. The proof follows from [Hi, Corol- 
lary 4.3]. Theorem 4 (ii) is also true (assuming the continuum hypothesis). The only substantive change that is necessary to complete the proof is to use either the Theorem of this paper or [HV-2, Theorem 3] to obtain a $(\mathfrak{A}, \mathcal{C})$-measurable selector for $K \cap T$.

\section{REFERENCES}

[C] C. Castaing, Sur les multi-applications mesurables, Revue Française d'Informatique et de Recherche Operationnelle, 1 (1967), pp. 91-126.

[Hi] C. J. Himmelberg, Measurable relations, Fund. Math., 87 (1975), pp. 53.72.

[Hu] S. T. Hu, Theory of Retracts, Wayne State University Press, Detroit (1965).

[HPV] C. J. Himmelberg - T. Parthasarathy - F. S. Van Vlecck, On measurable relations, Fondamenta Mathematicae, to appear.

[HV-1] C. J. HIMmelberg - F. S. VAN VLECK, Multifunctions on abstract measurable spaces and applications to stochastio decision theory, Annali Mat. Pura Appl., 101 (1974), pp. 229-236.

[HV-2] C. J. Himmeliberg - F. S. VAN VLeCK, Some selection theorems for measurable funetions, Canad. J. Math., 21 (1969), pp. 394-399.

[K] J. L. KeLLey, General Topology, D. Van Nostrand Co., Now York (1955).

[N] Togo Nishidra, Two counterexamples for measurable relations, Rocky Mountain J. Math., to appear.

[W] D. H. WAGNER, Survey of measurable selection theorems, SIAM J. Control and Optimization, 15 (1977), pp. 859-903. 